\title{
Physicochemical Characterization of Sulfated Polysaccharides from Green Seaweeds: Ulva pertusa and Ulva conglobata
}

\author{
Masako Yamamoto, Yuji Tadokoro*, Hiroshi Imai \\ and Kiyo MiTA \\ Department of Home Economics, Hiroshima Women's University, Hiroshima 734 \\ and *Department of Chemistry, Faculty of Integrated Art \\ and Sciences, Hiroshima University, Hiroshima, Japan
}

Received July 11, 1979

\begin{abstract}
Sulfated polysaccharides extracted from Ulva pertusa (UP) and Ulva conglobata (UC) were purified on DEAE-cellulose and Sepharose 4B gel columns. Both polymers were confirmed to be homogeneous by gel-filtration chromatography and ultracentrifugation. They were composed of rhamnose, xylose, glucuronic acid, glucose, galactose and sulfate. UP contained a high level of glucuronic acid $(27 \%)$ and UC, a low level $(6 \%)$, and galactose was not detected in UP. A sedimentation equilibrium measurement showed distinctly different molecular weights: $9.1 \times 10^{4}$ for UP and $8.2 \times 10^{5}$ for UC, and these results were in good agreement with values estimated by gel chromatography. Specific optical rotations for UP and UC also differed at, $[\alpha]_{\mathrm{D}}^{20}=-77.8^{\circ}$ and $-26.3^{\circ}$, respectively. In spite of these differences, the two polymers showed similar physicochemical behavior and had similar random coil conformation in acidic buffer solutions.
\end{abstract}

Taxonomy of mucilages in seaweeds is not yet established on a sound basis. It has been attempted by the use of biological, chemical and mixed methods, ${ }^{1 \sim 3\}}$ but in many cases the fundamental chemical structures of polyelectrolytic components in mucilage are still much less explored than other biological macromolecules such as some proteins and RNA's. Percival et al ${ }^{47}$ classified the mucilages from different sources on a biological basis, while the taxonomical interrelation between their physicochemical properties and biological species is also undoubtedly important to the development of molecular evolution.

In this connection we intended to establish the molecular taxonomy of mucilages in seaweeds from a physicochemical viewpoint. Little research has been made on the intact macromolecular entity of green seaweeds, compared with that on their fragmented components. It is important for molecular clarification of the mucilages to separate and purify polyelectrolytic polysaccharides in their intact form. In this work the sulfated polysaccharides from Ulva pertusa (UP) and Ulva conglobata (UC) were purified by fractionation on
DEAE-cellulose and Sepharose 4B columns and subjected to measurement of sedimentation and viscometric analyses.

\section{EXPERIMENTAL}

Raw Materials. UP was collected in Ujina Bay, Hiroshima, and UC i Karatsu Bay, Saga, in the spring of 1974. One kilogram of finely disrupted seaweeds was used for extraction of polysaccharide components with hot water (about $90^{\circ} \mathrm{C}, 20$ volumes). Crude mucilages were obtained from the extracts after dialysis against running tap water. The mucilages were further separated into sulfated polysaccharides and neutral ones by the method of iodine complexation. ${ }^{5)}$ The yields of the crude sulfated polysaccharides were $18.6 \mathrm{~g}$ and $11.1 \mathrm{~g}$ from UP and UC, respectively, while the neutral one was obtained only from UP in a yield of $0.3 \mathrm{~g}$.

Fractionation. Half a gram of the crude sulfated polysaccharides was dissolved in distilled water $(10 \mathrm{ml})$, and then the solution was applied to a DEAE-cellulose column $(3 \times 30 \mathrm{~cm})$ conditioned previously. Elution was performed stepwise with $500 \mathrm{ml}$ each of $0.5,1.0$, 1.5 and $2.0 \mathrm{M}$ aqueous potassium chloride solutions. Most of the polysaccharides were eluted with the $1.0 \mathrm{M}$ potassium chloride solution which was dialyzed at room temperature for $24 \mathrm{hr}$ against running tap water and then evaporated at $40^{\circ} \mathrm{C}$. After precipitation with 
absolute alcohol (10 volumes), the polysaccharides were completely dried in vacuum. The dried materials were further purified by repetition of gel-filtration chromatography; a column $(2.5 \times 62 \mathrm{~cm})$ packed with Sepharose 4B (Pharmacia Fine Chemicals) was pre-equilibrated with a $0.1 \mathrm{M}$ citrate buffer of pH 3.2 (5 volumes). ${ }^{\text {b) }}$ Then, $1 \%$ polysaccharide solution $(2 \mathrm{ml})$ was eluted with the same buffer, every $10 \mathrm{ml}$ of the eluate being collected. The polysaccharide contents in each fraction were determined by the phenol-sulfuric acid method.7) The profile of the first elution showed the presence of a small shoulder on the frontal side of the main peak. After three repeated runs to remove minor components, the final profile gave a single sharp peak. Of course, the purified samples were confirmed to have undergone no decomposition under the extracting conditions mentioned elsewhere. ${ }^{8)}$ All the subsequent analyses, physical and chemical, were done on the samples. Furthermore the determination of the molecular weight was carried out on the same column $(2.5 \times 62 \mathrm{~cm})$ with Sepharose $4 \mathrm{~B}$ and the same eluent at a flow rate of $10 \mathrm{ml} / \mathrm{hr}$, every $5 \mathrm{ml}$ of the eluate being collected. Dextran T10, T20, T70, T150 and T500 were used as standards.

General Analyses, Uronic acid contents were analyzed by the modified carbazol-sulfuric acid method, ${ }^{, 7}$ and sulfate contents by the modified Schöniger's method. ${ }^{10}$ Optical rotation of sugars was measured with a Hitachi $\mathrm{PO}-\mathrm{B}$ polarimeter at $20^{\circ} \mathrm{C}$.

After hydrolysis, determination of sugar composition of the samples was carried out: they were completely hydrolyzed with $1 \mathrm{~N}$ (aqueous) sulfuric acid at $95 \sim 100^{\circ} \mathrm{C}$ for $4 \mathrm{hr}$ and then neutralized with $1 \mathrm{M}$ barium hydroxide solution. After filtration, the hydrolyzates were concentrated to a syrup under reduced pressure. A portion of this syrup was converted into alditol acetate derivatives ${ }^{11}$ for gas chromatography (g. 1. c.).

Chromatographic analyses of the samples on a Hitachi K53 gas chromatograph equipped with a FID detector were carried out at $200^{\circ} \mathrm{C}$ by flowing nitrogen gas (mobile phase) at a rate of $20 \mathrm{ml} / \mathrm{min}$ into a stainless steel column $(0.3 \times 100 \mathrm{~cm})$, packed with Neopack $1 \mathrm{~A}$ coated with $3 \%$ ECNSS-M (stationary phase). Relative concentrations of monomer sugars in the hydrolyzates were determined in g.l.c. peak areas, which were calibrated for the standard solutions of five possible monosaccharides, rhamnose, xylose, mannose, galactose, glucose and arabinose, in the range of $0 \sim 10 \mathrm{mg}$ / $\mathrm{ml}$.

Viscometry. The samples were dissolved in both water and $0.1 \mathrm{~m}$ citrate buffer of $\mathrm{pH} 3.2$ at $1 \%$ concentration: the former was used to obtain information on characteristic behavior of a poly-electrolyte in the absence of electrolytes of low molecular weight. After the solution was filtered through sintered glass of $\mathrm{G} 3$, measurement was carried out in the solution at $20^{\circ} \pm$ $0.01^{\circ} \mathrm{C}$ with a capillary viscometer of Ubbelohde type, whose flow time for water was 120 .

Ultracentrifugation. Fifty milligrams of the samples were dissolved into $0.1 \mathrm{M}$ citrate buffer $(10 \mathrm{ml})$, and the solutions were dialyzed against the same buffer at room temperature overnight. The dialyzed solutions were used in sedimentation measurement, and the dialyzates were also used as the solvent of optical reference. During dialysis, low molecular weight ions could be redistributed through membrane, and then the concentrations of macromolecular components should have changed to some extent. ${ }^{12)}$ Therefore, we redetermined their concentrations by the method mentioned above. ${ }^{7}$ )

Partial specific volumes, which are necessary for buoyant correction of the molecular weights determined, can be computed from density measurements at the temperature of equilibrium sedimentation runs. The apparatus of densimetry was a Seiko Density Meter 410 , calibrated by the use of aqueous potassium chloride. The densities were $\rho=1.01049$ and $1.01005 \mathrm{~g} /$ $\mathrm{ml}$ at concentrations of $c=4.35 \times 10^{-3}$ and $2.97 \times$ $10^{-3} \mathrm{~g} / \mathrm{ml}$ for UP and UC, respectively, while solvent density was determined to be $\rho_{0}=1.0090 \mathrm{~g} / \mathrm{ml}$. Then the partial specific volume $\bar{v}$ can be computed by the following expression, ${ }^{13)}$

$$
\bar{v}=\frac{1}{\rho_{0}}\left(1-\frac{\rho-\rho_{0}}{c}\right)
$$

Thus the values of $\bar{v}$ were estimated to be 0.64 and $0.63 \mathrm{ml} / \mathrm{g}$ for $\mathrm{UP}$ and $\mathrm{UC}$, respectively, at $10^{\circ} \mathrm{C}$.

Measurement of sedimentation was made with a Hitachi 282 analytical ultracentrifuge, and the concentration distributions were taken by schlieren and interference optics. Optical patterns on the plates were read off with a Nikon projector within a tolerance of $\pm 2 \times 10^{-3} \mathrm{~mm}$. The sedimentation velocity was measured at a rotor speed of $60,000 \mathrm{rpm}$ at $20^{\circ} \mathrm{C}$ by the use of schlieren optics and a double sector cell. Equilibrium sedimentation was carried out at $10^{\circ} \mathrm{C}$ to prevent the putrefaction of the samples by the use of multichannel cell with interference optics at a rotor speed of 6000 to $12,000 \mathrm{rpm}$. All the runs of equilibrium sedimentation were continued for at least $50 \mathrm{hr}$ with a short length of solution column (about $0.3 \mathrm{~cm}$ ) in the cell. Refractive index increments of the samples were determined in the same buffer of $0.1 \mathrm{M}$ citrate at a rotor speed of $1000 \sim 2000 \mathrm{rpm}$ by the use of a synthetic boundary cell of capillary type, which also gave interference patterns allowing calculation of diffusion coefficients for the macromolecular components in solutions.

\section{RESULTS AND DISCUSSION}

The specific optical rotations and the con- 
Table I. Analytical Results of the Main Fractions of Sulfated Polysaccharides

\begin{tabular}{lcccccccc}
\hline \multirow{2}{*}{ Sample } & \multirow{2}{*}{$\begin{array}{c}{[\alpha]_{\mathrm{D}}^{20}} \\
\text { water }\end{array}$} & $\begin{array}{c}\text { Sulfate } \\
(\%)\end{array}$ & & \multicolumn{5}{c}{ Sugar component $(\%)^{b}$} \\
\cline { 7 - 9 } & & & & Rhamnose & Xylose & Galactose & Glucose & $\begin{array}{c}\text { Glucuronic } \\
\text { acid }\end{array}$ \\
\hline U. pertusa & $-77.8^{\circ}$ & 12.0 & 51 & 21 & 0 & 1 & 27 \\
U. conglobata & $-26.3^{\circ}$ & 11.9 & 57 & 20 & 5 & 12 & 6 \\
\hline
\end{tabular}

Specific optical rotation was measured at a concentration of 0.141 for UP or 0.135 for UC.

a Sulfate content is indicated in weight percentage for the sulfated polysaccharides.

$b$ Each sugar's is in molar percentage for carbohydrate.

tents of both sulfate and sugars of sulfated polysaccharides (SPS) from UP and UC are listed in Table I, where the sums of the numerical values of sugar components are adjusted to $100 \%$ on a molar basis. Both SPS's were mainly composed of rhamnose and xylose, while glucuronic acid for UP seems to be partially substituted by galactose and glucose for UC. On the other hand, a remarkable difference in specific rotation between the two is shown in Table I. Although it is believed that the difference does not simply correspond to the composition of sugars, overall optical rotations of polysaccharide could be controlled by way of $\alpha$ - or $\beta$-linkages between successive monomer sugars and local sequence of $\mathrm{L}$ - and $\mathrm{D}$ forms of monomer in skeletal chains. ${ }^{141}$

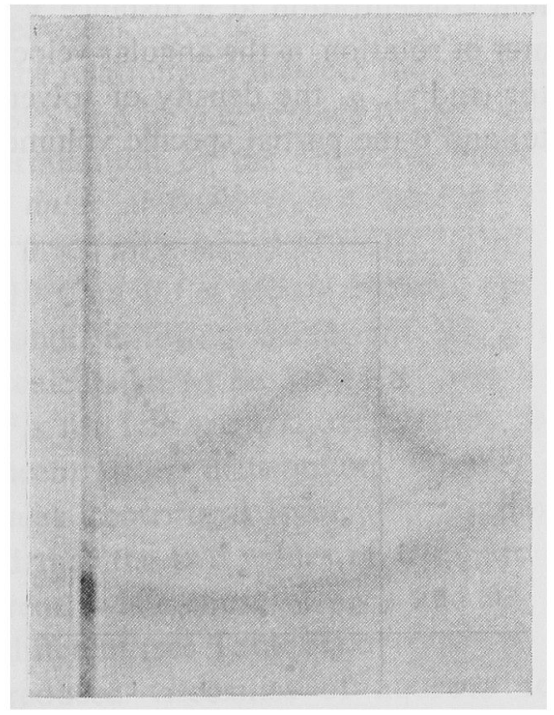

(1)
The molecular homodispersity of the samples was examined by ultracentrifugation and gelfiltration chromatography. By either method both SPS's showed a single specific peak as shown in Figs. 1 and 2, the existence of one component being confirmed from the experimental criteria. On the other hand, the sedimentation velocities of moving boundary (Fig. 1) for the SPS's were measured at their various concentrations: from the results we obtained the sedimentation coefficients, $s_{20, \mathrm{w}}$, which were used in later consideration. The values of $s_{20, \mathrm{w}}$ decreased with an increase in concentration, and the extrapolated values to infinite dilution $^{15)}$ were estimated to be $s_{20, \mathrm{w}}^{0}=3.5$ and $11.5 \mathrm{~S}$ for UP and UC, respectively.

The molecular weights of SPS's were esti-

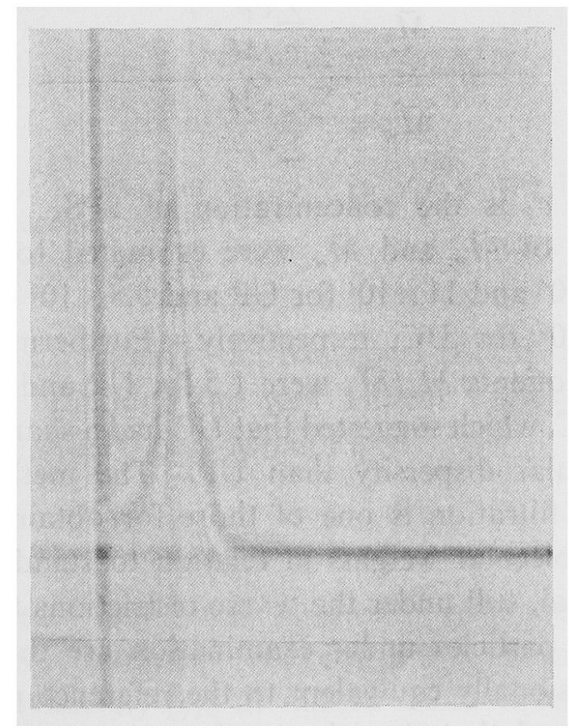

(2)

FIG. 1. Sedimentation Patterns of the Sample in Ultracentrifugation.

Rotor speed: $60,000 \mathrm{rpm}$, temperature: $20^{\circ} \mathrm{C}$. (1) $0.158 \%$ UP at $72 \mathrm{~min}$, angle of the schlieren diagram: $70^{\circ}$. (2) $0.300 \% \mathrm{UC}$ at $25 \mathrm{~min}, 80^{\circ}$. 


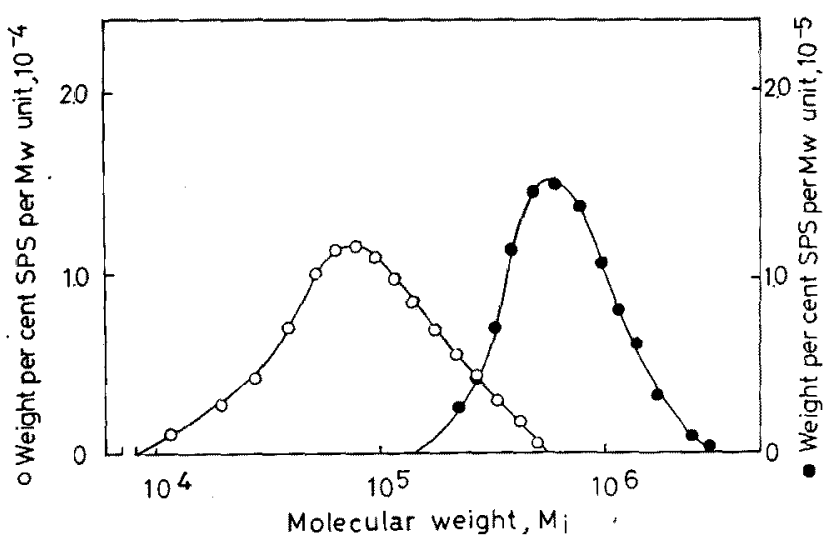

FIG. 2. Molecular Weight Distribution Curves by Gel-filtration Chromatography on Sepharose $4 \mathrm{~B}$.

(O): UP, (•): UC.

mated by gel filtration and equilibrium sedimentation. In the former procedure, the molecular weights, $M_{i}$, were estimated from the elution volume data of the obscissa of the elution diagram of SPS on a calibrated gel column. Then the molecular weight distribution of SPS's was obtained as shown in Fig. 2, from which the average molecular weights were calculated by the following equations,

$$
\begin{aligned}
\bar{M}_{n} & =\frac{\sum c_{i}}{\sum c_{i} / M_{i}} \\
\bar{M}_{w} & =\frac{\sum c_{i} \cdot M_{i}}{\sum c_{i}}
\end{aligned}
$$

where $c_{i}$ is the concentration of SPS. The values of $\bar{M}_{n}$ and $\bar{M}_{w}$ were estimated to be $6.9 \times 10^{4}$ and $11 \times 10^{4}$ for UP and $5.8 \times 10^{5}$ and $7.7 \times 10^{5}$ for UC, respectively. Furthermore the quotients $\bar{M}_{n} / \bar{M}_{w}$ were 1.5 for UP and 1.3 for UC, which suggested that UC had a sharper molecular dispersity than UP. The method of gel filtration is one of those for obtaining the molecular weights in relation to standard material, still under the severe restrictions that solute particles under examination are threedimensionally equivalent to the reference particles and that they do not have any specific interactions with gel phase. ${ }^{16)}$ In the present cases of sulfated polysaccharides, high rigidity of the skeletal chains ${ }^{17}$ might render them less internal rotation about bonds between two successive monomer sugars. Therefore, we would like to obtain the molecular weights at a thermodynamic (sedimentation) equilibrium. The apparent molecular weight, $M_{a p p}$, was calculated from equilibrium distribution of the concentration, ${ }^{15)}$

$$
M_{a p p}=\frac{2 R T}{\left(1-v \rho_{0}\right) \omega^{2}} \frac{d \ln c(x)}{d(x)^{2}}
$$

where $\ln c(x)$ is the logarithm of the equilibrium concentration at a distance $x$ from the center of rotation, $\omega$ the angular velocity of the rotor $(\mathrm{rad} / \mathrm{s}), \rho_{0}$ the density of solvent (dialyzate) and $\bar{v}$ the partial specific volume of SPS.

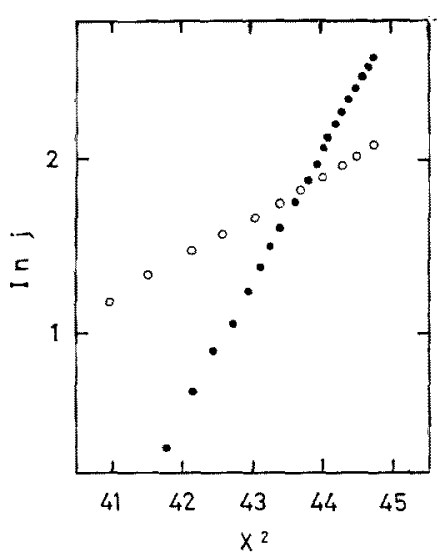

FIG. 3. Plots of in $J$ vs. $x^{2}$. (O): UP, (O): UC. 


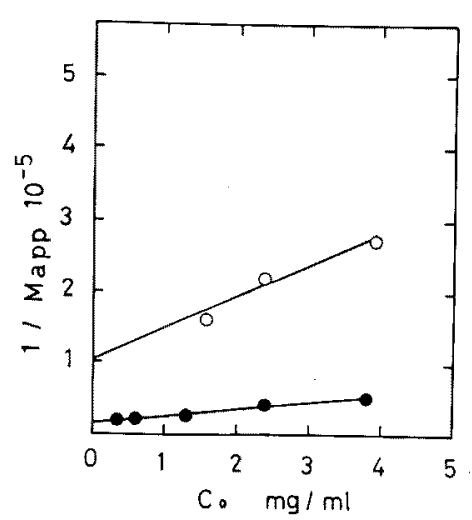

FlG. 4. Plots of $1 / M_{a p p} v s$, the Origin Concentration, $c_{0}$.

(O): UP, (⿶): UC.

Plots of $\ln c(x)$ vs. $x^{2}$ were curved upward for both SPS's as can be seen in Fig. 3, meaning possible thermodynamic nonideality of the solutions. Hence, one can estimate the true molecular weight $M_{0}$ on equilibrium experiments at different initial concentrations, ${ }^{18)}$ and the values of $M_{0}$ were determined to be $(9.1 \pm$ $0.8) \times 10^{4}$ for UP and $(8.2 \pm 0.7) \times 10^{5}$ for $U C$ by extrapolation $c_{0}=0$ in Fig. 4 . These values are in good agreement with the ones from the gel filtration, which were approximately as large as those of sulfated polysaccharides of brown seaweeds reported previously. ${ }^{19,20}$,

Now the relationship between the concentration of SPS and its interference fringe ${ }^{21)}$ led to the determination of the refractive index increment $d n / d c$ at $0.099\left(c_{0}=4.304 \times 10^{-3}\right)$ for $\mathrm{UP}$ and $0.100 \mathrm{ml} / \mathrm{g}\left(c_{0}=2.970 \times 10^{-3} \mathrm{~g} / \mathrm{ml}\right)$ for $\mathrm{UC}$ at $10^{\circ} \mathrm{C}$ in $0.1 \mathrm{M}$ citrate buffer. On the other hand, diffusion coefficient $D_{20, \mathrm{w}}$ can also be calculated to be $2.4 \times 10^{-7}$ and $1.4 \times$ $10^{-7} \mathrm{~cm}^{2} / \mathrm{s}$ for UP and UC, respectively, from the concentration distribution in the cell under weak centrifugal force. ${ }^{22,23)}$ It should be noted that the two values of $D_{20 \text {, w }}$ are not so different, while those of $s_{20, w}$ and $M_{0}$ are greatly different (see Table II).

The reduced viscosities of aqueous solutions of both SPS's without buffer electrolyte in Fig. 5 show typical behavior of polyelectrolyte viscosity where the reduced viscosity sharply increases with a decrease in concentra-

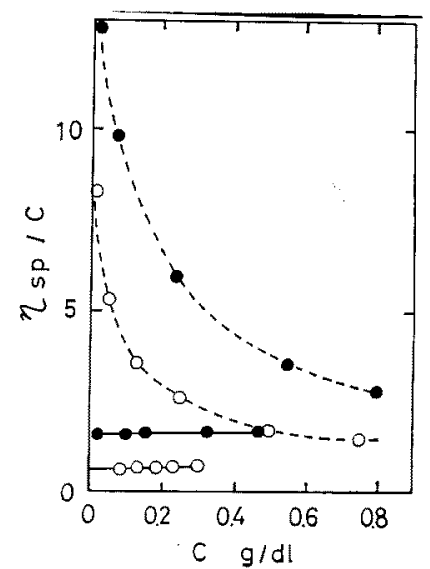

FIG. 5. The Concentration-dependence of the Reduced Viscosity in Aqueous Solution, Dashed line, and in $0.1 \mathrm{M}$ Citrate Buffer, Solid line.

(O): UP, (•): UC.

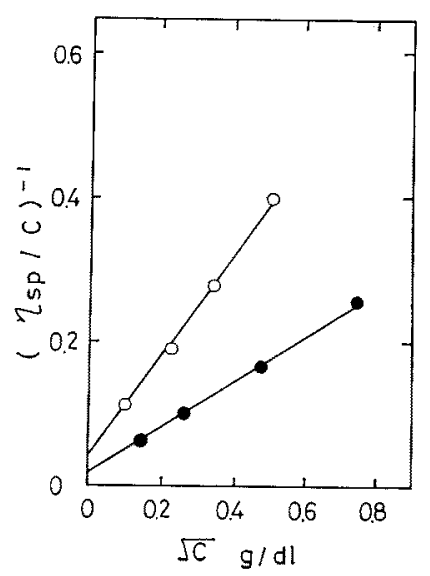

FIG. 6. Plots of $\left(\eta_{\mathrm{sp}} / c\right)^{-1} v s, \sqrt{\bar{c}}$ of the Data in Aqueous Solution.

(O): UP, (๑): UC.

tion. Actually the reciprocal plot of $\eta_{\mathrm{sp}} / c$ vs $\sqrt{c}$ followed the well known Fuoss-Strauss equation ${ }^{24)}$ as shown in Fig. 6.

$$
\left(\eta_{\mathrm{sp}} / c\right)_{\mathrm{water}}=\frac{A}{1+B \sqrt{c}}
$$

where $c$ is the concentration in $\mathrm{g} / \mathrm{dl}$, and $A$ and $B$ are constants. The term $A$ is equal to intrinsic viscosity $[\eta]_{w}$ at infinite dilution and then the values were estimated to be 25 and $44 \mathrm{dl} / \mathrm{g}$, respectively, for UP and UC, which are consistent with the molecular weights. On the other hand, addition of the buffer solutes to the 
Table II. Summary of the Physicochemical Properties

\begin{tabular}{lcc}
\hline \multicolumn{1}{c}{ Property } & U. pertusa & U. conglobata \\
\hline Sedimentation coefficient $\left(s_{20, \mathrm{w}}\right), 10^{-13} \mathrm{~s}$ & 3.5 & 11.1 \\
Diffusion coefficient $\left(D_{20, \mathrm{w}}\right), 10^{-7} \mathrm{~cm}^{2} / \mathrm{s}$ & 2.4 & 1.4 \\
Partial specific volume $(\bar{v}), \mathrm{cm}^{3} / \mathrm{g}$ & 0.64 & 0.63 \\
Differential refractive index increment $(d n / d c), \mathrm{ml} / \mathrm{g}$ & 0.099 & 0.10 \\
Molecular weight by ultracentrifugation $\left(M_{0}\right), 10^{5}$ & 0.91 & $8: 2$ \\
Molecular weight by gel chromatography $\left(M_{w}\right), 10^{5}$ & 1.1 & 7.7 \\
Limiting viscosity number $[\eta]_{20}, \mathrm{~g} / \mathrm{dl}$ & 0.51 & 1.46 \\
Limiting viscosity number $\mathrm{A}_{\mathrm{H}_{2} \mathrm{o}}, \mathrm{g} / \mathrm{dl}$ & 25 & 44 \\
\hline
\end{tabular}

SPS aqueous solutions changed the curves into a linear form and depressed the intrinsic values $[\eta]$ to 0.51 for UP and $1.46 \mathrm{dl} / \mathrm{g}$ for UC. Also the values of an expansion factor ${ }^{25)} \alpha_{\eta}$ obtained from these viscosity data, the factor being defined to be $\alpha_{\eta}=\left([\eta]_{w} /[\eta]\right)^{1 / 3}$, were computed to be $3.5\left(=(25 / 0.51)^{1 / 3}\right)$ and $3.1\left(=(44 / 1.46)^{1 / 3}\right)$ for UP and UC, respectively. The difference between the two were too small to explain the two SPS's as linear polymers. In addition, the small values of $[\eta]$ in comparison with $[\eta]=5.08 \mathrm{dl} / \mathrm{g}$ of hyaluronate ${ }^{26)}\left(M=3 \times 10^{5}\right)$, linear polyelectrolyte, also suggest that the SPS's may have a branching structure which is not so significant as glycogen ${ }^{27)}$ because of their considerable expansion. Indeed, Percival et al has reported that the sulfated polysaccharide of Cladophora rupestris, green seaweed, would be branched. ${ }^{28}$

Table II lists all the results from the present physicochemical measurement of UP and UC. The partial specific volumes and refractive index increments, which correspond more closely to specific quantities (that is, physical quantities pertaining to unit extensive properties) than to macromolecular entity as a whole, are identical with respect to both $\mathrm{Ul}$ vaceas. Therefore, this means that the SPS of neither UP nor UC has any special higherorder conformation in solution. In the widest physical sense, for example, the values of $d n / d c$ depend on the average number of electrons in a unit volume of the systems. Now, the values of 0.099 and 0.100 were very small compared with other polysaccharides, namely, $0.152,0.150$ and $0.143 \mathrm{ml} / \mathrm{g}$ for amylose $_{,}{ }^{29)}$ amylopectin ${ }^{27)}$ and hyaluronate, ${ }^{26)}$ (respectively,) which also suggest that SPS's may have much expanded conformations. When we compute the value of

$$
\beta=\Phi^{1 / 3} P^{-1}=s^{0}[\eta]^{1 / 3} \gamma_{0} N_{A} /\left(1-\bar{v} \rho_{0}\right) M^{2 / 3}
$$

on the basis of the modern theory of polymer solution, ${ }^{30}$ they take the same values of $2.4 \times$ $10^{6}$ for UP and UC. The value of $\beta$ indicates both SPS's to be random coil in solution, and the much expanded structure of SPS's should enable them to exhibit characteristics of random coil, even if they have some branched points.

Some physicochemical properties of the SPS's such as molecular weight, intrinsic viscosity, sedimentation coefficient and diffusion coefficient differed from one to another, while the two SPS's might be considered to have the identical molecular conformation in solution. These discrepancies in physicochemical properties of the two species cannot be directly correlated to the biological species on the basis of the present work alone. However, it is considered that the two sulfated polysaccharides from UP and UC, Ulvaceas, have similar random coil conformations in buffer solution in spite of their different molecular weights.

\section{REFERENCES}

1) R. L. Whistler and W. M. Corbett, "The Carbohydrates," Academic Press, New York, N.Y., 1957, p. 641.

2) R. L. Whistler and C. I. Smart, "Polysaccharide Chemistry," Academic Press, New York, N. Y., 1953.

3) G. O. Aspinall, "Polysaccharides," Pergamon Press, London, 1970.

4) E. Percival and R. H. McDowell, "Chemistry and Enzymology of Marine Algal Polysaccharide," Academic Press, New York, N. Y., 1967, p. 2. 
5) J. P. McKinnell and E. Percival, J. Chem. Soc., 3141 (1962).

6) Usually, polyacid such as polyglutamic acid in solution might be precipitated in their protonated forms. However, sulfated polysaccharides are completely different in precipitation behavior in relation to pH's of the solutions. Therefore, all of the present works were done at a rather low pH of 3.2 .

7) M. Dubois, K. A. Gilles, J. K, Hamilton, P. A. Rebers and F. Smith, Anal. Chem., 28, 350 (1956).

8) M. Yamamoto, U. Tadokoro and K. Mita, manuscript in preparation.

9) T. Bitter and H. M. Muir, Anal. Biochem., 4, 330 (1962).

10) S. Ohta, Bull. Anal. Chem., 17, 1322 (1968).

11) J. S. Sawaldekan, H. Slonek and A. Jeaner, Anal. Chem., 37, 1603 (1965).

12) E. E. Casassa and H. Eisenberg, $A d v$. Protein Chem., 19, 287 (1964).

13) O. Katky, H. Leopold and C. W. Schmid, Methods in Enzymology, 27D, 98, (1973).

14) C. S. Hudson, J. Am. Chem. Soc., 31, 66 (1909); 52, 1680, 1707 (1930).

15) T. J. Bowen, "An Introduction to Ultracentrifugation," Wiley Intersciences, London, 1970, p. 54 .

16) K. A. Granath and B. E. Kuist, J. Chromatog., 28,
69 (1967).

17) Th. Hohn and H. Schaller, Biochim. Biophys. Acta, 138, 466 (1967).

18) H. Fujita, "Foundation of Ultracentrifugal Analysis," John Wiley \& Sons, Inc., New York, N. Y., 1975, p. 284.

19) L. E. Cepnnko et al., Izvestiia Bysshikh Uchebnykh Zavedenit (P. T.), 27 (1975).

20) G. Bernardi and G. F. Springer, J. Biol. Chem., 237, 75 (1962).

21) E. E. Casassa and H. Eisenberg, Advances in Protein Chemistry, 19, 287 (1964).

22) M. Nagasawa and H. Fujita, J. Am. Chem. Soc., 86, 3005 (1964).

23) Tables of the Error Function and its Derivative, National Bureau of Standards, Washigton, D. C., 1954.

24) R. M. Fuoss, J. Polymer Sci., 3, 603 (1948).

25) A. Oth and P. Doty; J. Phys. Chem., 56, 43 (1952).

26) R. L. Cleland, Biopolymers, 6, 1519 (1968).

27) W. Banks, R. Geddes, C. T. Greenwood and G. Hones, Stärke, 24, 245 (1972).

28) E. J. Bourne, P. G. Johnson and E. Percival, $J$. Chem. Soc., 1970, 1561.

29) M. Kodama and H. Noda, Biopolymers, 17, 985 (1978).

30) L. Mandelkern and P. J. Flory, J. Chem. Phys, 20, 212 (1952). 